\title{
Structural determinants of long-term functional outcomes in young children with cystic fibrosis
}

\author{
Lidija Turkovic ${ }^{1}$, Daan Caudri ${ }^{1,2,3}$, Tim Rosenow (1) ${ }^{1,4}$, Oded Breuer (1) 1 , \\ Conor Murray ${ }^{5}$, Harm A.W.M. Tiddens ${ }^{3}$, Fiona Ramanauskas ${ }^{6}$, Sarath \\ C. Ranganathan ${ }^{6,7,8}$, Graham L. Hall ${ }^{1,9}$ and Stephen M. Stick ${ }^{1,2,4}$, on behalf of \\ AREST CF
}

Affiliations: ${ }^{1}$ Telethon Kids Institute, Perth, Australia. ${ }^{2}$ Dept of Respiratory and Sleep Medicine, Perth Children's Hospital, Perth, Australia. ${ }^{3}$ Dept of Pediatrics/Respiratory Medicine, Erasmus University, Rotterdam, The Netherlands. ${ }^{4}$ Centre for Child Health Research, University of Western Australia, Perth, Australia. ${ }^{5}$ Dept of Diagnostic Imaging, Princess Margaret Hospital, Perth, Australia. 'Murdoch Children's Research Institute, Parkville, Australia. ${ }^{7}$ Dept of Respiratory and Sleep Medicine, Royal Children's Hospital, Parkville, Australia. ${ }^{8}$ Dept of Pediatrics, University of Melbourne, Parkville, Australia. ${ }^{9}$ School of Physiotherapy and Exercise Science, Curtin University, Perth, Australia.

Correspondence: Lidija Turkovic, Telethon Kids Institute, 15 Hospital Way, Nedlands, WA, 6009 Australia. E-mail: lidijat@gmail.com

@ERSpublications

Structural lung changes identified on a chest CT scan in children with cystic fibrosis under 6 years of age can identify those at risk of adverse long-term outcomes http://bit.ly/39QH5jv

Cite this article as: Turkovic L, Caudri D, Rosenow T, et al. Structural determinants of long-term functional outcomes in young children with cystic fibrosis. Eur Respir J 2020; 55: 1900748 [https://doi.org/ 10.1183/13993003.00748-2019].

\section{ABSTRACT}

Background: Accelerated lung function decline in individuals with cystic fibrosis (CF) starts in adolescence with respiratory complications being the most common cause of death in later life. Factors contributing to lung function decline are not well understood, in particular its relationship with structural lung disease in early childhood. Detection and management of structural lung disease could be an important step in improving outcomes in CF patients.

Methods: Annual chest computed tomography (CT) scans were available from 2005 to 2016 as a part of the AREST CF cohort for children aged 3 months to 6 years. Annual spirometry measurements were available for $89.77 \%$ of the cohort (167 children aged 5-6 years) from age 5 to 15 years through outpatient clinics at Perth Children's Hospital (Perth, Australia) and The Royal Children's Hospital in Melbourne (Melbourne, Australia) (697 measurements, mean \pm sD age $9.3 \pm 2.1$ years).

Results: Children with a total CT score above the median at age 5-6 years were more likely to have abnormal forced expiratory volume in $1 \mathrm{~s}\left(\mathrm{FEV}_{1}\right)$ (adjusted hazard ratio 2.67 (1.06-6.72), $\left.\mathrm{p}=0.037\right)$ during the next 10 years compared to those below the median chest CT score. The extent of all structural abnormalities except bronchial wall thickening were associated with lower $\mathrm{FEV}_{1} \mathrm{Z}$-scores. Mucus plugging and trapped air were the most predictive sub-score (adjusted mean change $-0.17(-0.26--0.07) \mathrm{p}<0.001$ and $-0.09(-0.14--0.04) \mathrm{p}<0.001$, respectively).

Discussion: Chest CT identifies children at an early age who have adverse long-term outcomes. The prevention of structural lung damage should be a goal of early intervention and can be usefully assessed with chest CT. In an era of therapeutics that might alter disease trajectories, chest CT could provide an early readout of likely long-term success. 


\section{Introduction}

Cystic fibrosis (CF) is the most common life-shortening autosomal recessive disease affecting children, with an estimated incidence of one in 2500 live births in Caucasian populations [1]. Spirometry, an objective measure of lung function, is the most universally performed and accepted clinical test by which a patient's respiratory status, progress or decline is assessed. Forced expiratory volume in $1 \mathrm{~s}\left(\mathrm{FEV}_{1}\right)$ is the key clinical efficacy measure and driver in the definition of disease stage and treatment decisions, as a U.S. Food and Drug Administration approved primary outcome measure for clinical trials and in the regulatory approval of respiratory therapies in CF $[2,3]$.

Spirometry is usually not performed in children aged $<6$ years with CF and it commonly remains within the range of values in healthy children from 6 years of age until early adolescence, limiting its usefulness for assessing both clinical status and effects of interventions in early childhood. Hence, there is a great need for a biomarker that can be easily measured in early life and that can predict longer term outcomes.

Australian and international CF registries usually report spirometry data as "annual best" from the age of 6 years [4-6]. These data have shown that median percent predicted $\mathrm{FEV}_{1}$ increases in successive cohorts. However, when assessing $\mathrm{FEV}_{1}$ population trajectories, cohorts with a lower median $\mathrm{FEV}_{1}$ at age 6 years tend to maintain lower $\mathrm{FEV}_{1}$ throughout life with little change in the rate of the decline. This highlights that early childhood represents an important developmental period when intervention strategies could potentially prevent the early onset of lung damage and thereby have beneficial long-term effects for patients with CF. Standard spirometry fails to detect changes in structural lung disease [7]. Moreover, improvement in early lung function in successive cohorts has not prevented the significant decline that remains evident commencing during adolescence in CF $[6,8]$.

There is strong evidence that structural lung disease in CF begins in infancy, is progressive and is associated with inflammation and infection [9-11], frequency of pulmonary exacerbations and reduced quality of life $[12,13]$. Chest computed tomography (CT) is considered the "gold standard" for demonstrating CF structural lung disease.

One of the challenges in using chest CT for assessing clinical status, or as a potential surrogate end-point in early intervention trials, is trying to assess its relationship with traditional outcomes and anticipating what long-term effects might be expected from an intervention in early life when lung damage is relatively mild. The majority of published work has typically focused on comparisons tracking disease progression using spirometry and CT in school-aged or older children and adults [7, 14-16]. The relationships between the commonly observed CT-defined structural lung abnormalities in young children and long-term functional outcomes are not known.

Data were available from the Australian Respiratory Early Surveillance Team for Cystic Fibrosis (AREST CF) longitudinal paediatric cohort and routine outpatient clinic visits. We aimed to assess whether structural lung abnormalities in early childhood are predictive of lung function decline in the following 10 years of life and if so, to determine what the optimal age is to perform a CT scan in young children. These issues are of significant clinical importance since solutions would allow for closer clinical monitoring of high-risk young children and provision of an informative surrogate to monitor the effects and aid the development of interventions to prevent structural disease and subsequent $\mathrm{FEV}_{1}$ decline.

\section{Methods}

A detailed description of methods is provided in the online supplement.

\section{Study population}

The AREST CF programme includes a comprehensive assessment of infants born with CF starting at 3 months and then annually until 6 years of age. Children are assessed at two paediatric CF centres: Perth Children's Hospital (PCH) formerly Princess Margaret Hospital for Children in Perth and the Royal Children's Hospital (RCH) in Melbourne (both Australia). The study was approved by the ethics committee of each institution and written consent from parents was obtained at enrolment and at each follow-up.

\section{CT and spirometry}

A volume controlled, volumetric (since 2007 in Perth and 2010 in Melbourne) or limited slice (prior to 2007 and 2010, respectively) chest CT scan was obtained at end inspiration (pressure-volume of the respiratory system $\left.P_{\mathrm{rs}}=25 \mathrm{cmH}_{2} 0\right)$ and end expiration $\left(P_{\mathrm{rs}}=0 \mathrm{cmH}_{2} \mathrm{O}\right)$ as described previously [11]. CT images were scored for the presence of structural lung disease using a simplified Brody CF-CT scoring method [10, 11].

Spirometry measurements performed during admissions or outpatient visits for the AREST CF cohort were obtained retrospectively from $\mathrm{PCH}$ and $\mathrm{RCH}$ patient records from 5 to 15 years of age. Spirometry 
was performed according to current American Thoracic Society/European Respiratory Society guidelines $[17,18]$. For the years where more than one measurement was available per child, the annual best was selected for use in the analysis.

\section{Statistical analyses}

Approximately $10 \%$ of potential follow-up spirometry measurements between 5 to 15 years of age were not included in the analysis. Measurements were missing either because children were too young to provide technically acceptable results or for other technical reasons the attempts to provide a measurement were unacceptable (see figure 1 for detailed missing data information). In this study we report spirometric variables $\left(\mathrm{FEV}_{1}\right.$, forced vital capacity $(\mathrm{FVC}), \mathrm{FEV}_{1} / \mathrm{FVC}$ and forced expiratory flow at $25-75 \% \mathrm{FVC}$ $\left.\left(\mathrm{FEF}_{25-75}\right)\right)$ as Z-scores as recommended by the Global Lung Initiative [19].

The first step was to determine the optimal age at which the chest CT scan was the most clinically informative and most predictive of future lung function. Children were divided into three categories: 12 years old; 3-4 years old; and 5-6 years old. Total CF-CT score in each age category was calculated as a sum of four sub-scores (trapped air, mucus plugging, bronchiectasis and bronchial wall thickening) and was divided into medians. While there were children that had a CT scan available in each age group, there was only one CT scan per child per age group. Observation time was defined as a 10-year period (515 years of age) and was of equal length for each age category. Lung function over time was assumed to follow a model of accelerated decline (i.e. with increasing age, the probability of children's lung function falling below defined Z-score threshold increases), therefore Weibull regression was used to calculate hazard ratios. For the ease of clinical interpretation, hazard ratios compared the time until $\mathrm{FEV}_{1} \mathrm{Z}_{\text {-scores }}$ fell below the lower limit of normal (i.e. -1.64 Z-scores) [20] for above versus below the median of total CF-CT score during the observation period (figure 2). Sensitivity analysis was also performed by including total CF-CT score as a continuous variable (instead of a binary variable) (eTable 8).

For the most predictive CF-CT score age, linear mixed effects models with random intercepts and slope subject effects and auto-regressive (lag 1) residual structure were fitted for spirometry Z-scores as continuous outcomes and CF-CT scores as exposures of interest (table 1, eTables 3, 4 and 5). Sensitivity analysis was also performed to asses if removal of participants that only had one or two follow-up spirometry measurements had an effect on the results (eTable 2). Body mass index (BMI) for age Z-score was modelled similarly as additional clinical outcome of interest (eTable 6). We performed additional sensitivity analyses by dividing participants into two groups by presence/absence of different sub-components and calculating the rate of decline in each group (eTable 7).

The percentage of variation in future $\mathrm{FEV}_{1}$ that was additionally explained by each structural sub-score was also assessed by fitting a series of multivariate cross-sectional regression models over a 5-year period. The results were compared with percentage of variation that was explained by earlier $\mathrm{FEV}_{1}$ measurement, i.e. percentage of variation explained by $\mathrm{FEV}_{1}$ at age 5-6 years in $\mathrm{FEV}_{1}$ outcomes at age 6-11 years (figure 3).

All model coefficients are presented unadjusted and adjusted for previously published confounding variables: homozygous Phe508del mutation, pancreatic insufficiency, sex, time elapsed between CT scan and spirometry, and test centre.

BMI for age Z-scores were calculated according to World Health Organization reference equations [21]. All analyses were performed using Stata v14 (Stata Statistical Software; StataCorp, College Station, TX, USA).

FIGURE 1 Percentage of missing spirometry data at each age group. In 2016, $94 \%$ of the AREST CF cohort that had computed tomography scans available at ages 5-6 years were < 15 years of age. The percentage of each given age is shown on the figure.

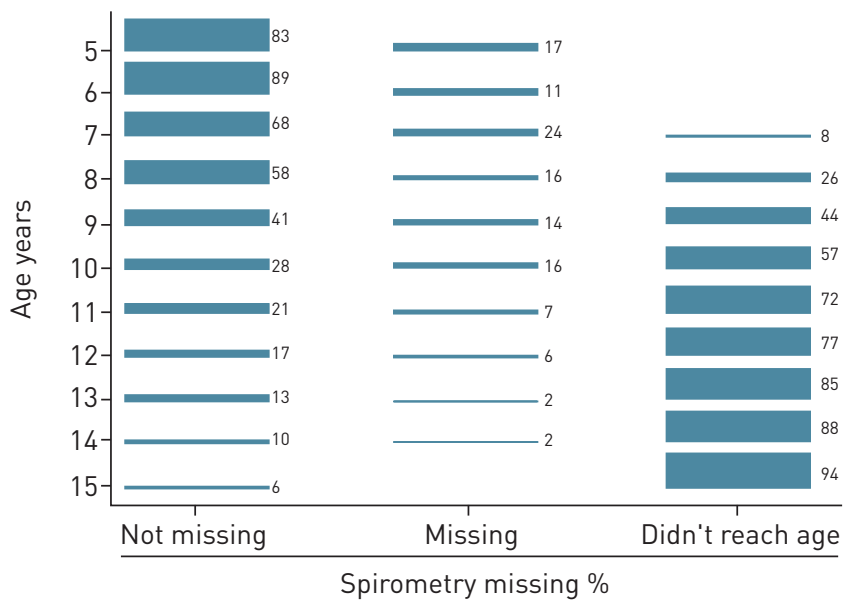




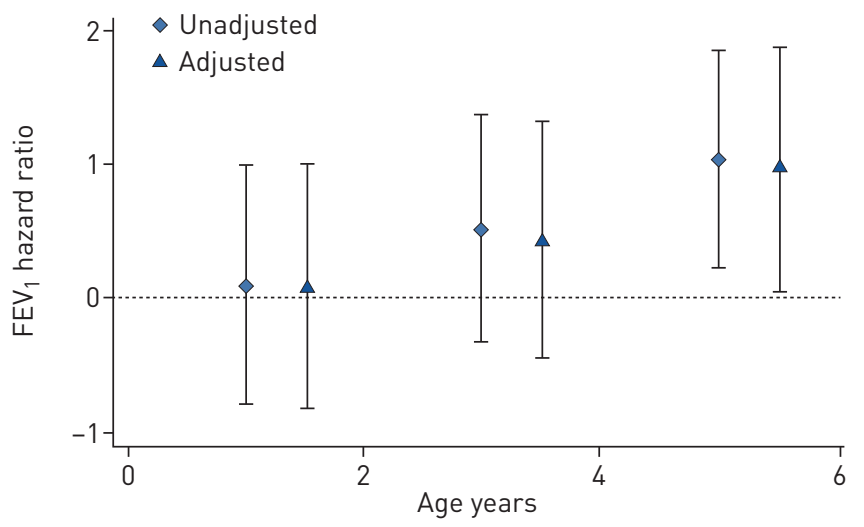

FIGURE 2 Adjusted hazard ratios of forced expiratory volume in $1 \mathrm{~s}\left(\mathrm{FEV}_{1}\right)$ falling below 1.64 Z-scores during the 10 years of observation with total cystic fibrosis-computed tomography score being above versus below the median at different ages. Hazard ratios were presented on the log scale. Cox regression was adjusted for intrinsic disease severity (homozygous D508 mutation, pancreatic sufficiency, meconium ileus and sex), test centre and time between computed tomography scan and spirometry.

\section{Results \\ Study population}

In the period that chest CT scans have been included in the follow-up a total of 325 children were included in the AREST CF cohort, with 196 children having at least one annual spirometry measurement between the age of 5 and 15 years. Of those 196 children, $48.47 \%$ were male with a mean \pm sD age of $3.67 \pm 0.21$ years during the AREST CF CT accrual period and 52.14\% were homozygous for the Phe508Del mutation. There was a total of 697 annual spirometry measurements available for the AREST CF cohort beyond the CT acquisition period from the combination of two sources $(\mathrm{PCH}$ and $\mathrm{RCH})$, with a mean $\pm \mathrm{SD}$ annual number of measurements per child being 4.79 \pm 3.36 . Of those 697 measurements, the mean \pm sD age of acquisition was 9.30 \pm 2.11 years. Mean \pm SD $\mathrm{FEV}_{1}$ and BMI for age Z-scores were $-0.07 \pm 1.23,0.44 \pm 0.90$ and $-1.11 \pm 1.57$, $-0.85 \pm 0.75$ at ages 5 years and at 15 years, respectively.

There was an increasing prevalence of structural lung disease over the first 6 years of life (eFigure 1a), most notably bronchiectasis (prevalence increased from $33.1 \%$ at age $1-2$ years to $73.7 \%$ at age 5-6 years) and mucus plugging (from $7.91 \%$ at age $1-2$ years to $37.13 \%$ at age 5-6 years). Mean $\mathrm{FEV}_{1}$ at age 5-6 years was within normal range with approximate start of decline at the age of 10-12 years (table 2 and eFigure 1b), while BMI for age Z-score showed slow but nearly linear decline over the 10 -year period (eFigure 1c).

\section{Determining the optimal age to perform a CT scan in early childhood}

During the 10-year observation period, $10.04 \%$ (70 out of 697) of annual spirometry measurements of $\mathrm{FEV}_{1}$ fell below the lower limit of normal threshold. Unadjusted and adjusted hazard ratios comparing the time until $\mathrm{FEV}_{1}$ fell below the threshold in children that had total CF-CT scores above versus below the

TABLE 1 Structural predictors of forced expiratory volume in $1 \mathrm{~s}$ Z-score decline

Unadjusted

\begin{tabular}{c}
\hline Mean change $(95 \% \mathrm{Cl})$ \\
697 \\
$-0.19(-0.29--0.09)$ \\
$-0.11(-0.16--0.05)$ \\
$-0.05(-0.09--0.01)$ \\
$-0.02(-0.07-0.03)$ \\
$-0.03(-0.05--0.01)$
\end{tabular}

Adjusted $^{\#}$

\begin{tabular}{cc}
\hline Mean change $(95 \% \mathrm{Cl})$ & $\mathrm{p}$-value \\
\hline $\mathbf{6 6 3}$ & $\mathrm{p}<0.001$ \\
$-\mathbf{0 . 1 7}(-\mathbf{0 . 2 6}--\mathbf{0 . 0 9 )}$ & $\mathrm{p}<0.001$ \\
$-\mathbf{0 . 0 9}(-\mathbf{0 . 1 4}--\mathbf{0 . 0 4 )}$ & $\mathrm{p}=0.051$ \\
$-0.04(-0.08-0.00)$ & $\mathrm{p}=0.734$ \\
$-0.01(-0.05-0.04)$ & $\mathrm{p}=\mathbf{0 . 0 0 6}$ \\
$-\mathbf{0 . 0 2}(-\mathbf{0 . 0 4}--\mathbf{0 . 0 1 )}$ &
\end{tabular}

Data presented for 167 children that had a computed tomography (CT) scan available at age 5-6 years and a total of 697 annual spirometry measurements in the following 10 years. CF: cystic fibrosis. ${ }^{\#}$ : models were adjusted for intrinsic disease severity (homozygous D508 mutation, pancreatic sufficiency and sex), test centre and age at spirometry. Bold data signifies $p<0.05$, mean change in CF-CT score of different structural components. 
TABLE 2 Clinical characteristics of children enrolled in AREST CF for which computed tomography scans were available

\begin{tabular}{|c|c|c|c|}
\hline & $1-2$ years & $3-4$ years & $5-6$ years \\
\hline Males & $70 / 139(50.36)$ & $81 / 162(50.00)$ & $82 / 167(49.10)$ \\
\hline \multicolumn{4}{|l|}{ Genotype } \\
\hline Homozygous Phe508del/Phe508del & $71 / 139$ (51.08) & $85 / 158(53.80)$ & $83 / 161(51.55)$ \\
\hline Heterozygous Phe508del/other & $64 / 139(46.04)$ & $68 / 158(43.04)$ & $71 / 161(44.10)$ \\
\hline PSA culture positive & $14 / 139(10.07)$ & $20 / 162(12.35)$ & $22 / 167(13.17)$ \\
\hline \multicolumn{4}{|l|}{ Structural lung disease } \\
\hline Bronchiectasis presence & 46/139 (33.09) & $98 / 162(60.49)$ & $123 / 167(73.65)$ \\
\hline Trapped air presence & $106 / 139(76.26)$ & $99 / 162(61.11)$ & $127 / 167(76.05)$ \\
\hline Mucus plugging presence & $11 / 139(7.91)$ & $44 / 162(27.16)$ & $62 / 167(37.13)$ \\
\hline Bronchial wall thickening presence & $106 / 139(76.26)$ & $142 / 162(87.65)$ & $154 / 167(92.22)$ \\
\hline FEF $_{25-75}$ Z-score & $-0.35 \pm 1.17$ & $-0.33 \pm 1.18$ & $-0.34 \pm 1.18$ \\
\hline
\end{tabular}

Data are presented as $\mathrm{n} / \mathrm{N}(\%)$ or mean $\pm \mathrm{SD}$. Scans were divided into age groups, with only a single scan per child in each age group. There are multiple scans of the same child in different age groups. PSA: Pseudomonas aeruginosa; FEV 1 : forced expiratory volume in $1 \mathrm{~s}$; FVC: forced vital capacity: $\mathrm{FEF}_{25-75}$ : forced expiratory flow at $25-75 \%$ of FVC.

median at different ages increased as children got older. At age 1-2 years adjusted hazard ratios (95\% CI) of time before $\mathrm{FEV}_{1} \mathrm{Z}$-score fell below -1.64 Z-scores was $1.09(0.43-2.76) \mathrm{p}=0.863$, at 3-4 years it was $1.55(0.63-3.78) \mathrm{p}=0.338$, and at age $5-6$ years children were nearly three times more likely to have their $\mathrm{FEV}_{1} \mathrm{Z}$-score fall below $-1.64(2.67(1.06-6.72), \mathrm{p}=0.037)$ if their total CF-CT score was above the median (figure 2).

Sensitivity analysis including a total CF-CT score as a continuous variable reaches the same conclusion and shows that for every point increase in CF-CT score, the adjusted hazard ratio increased by (1.06 $(1.02-1.11) \mathrm{p}=0.007)$ (eTable 8).

\section{Prediction of lung function trajectory based on different structural abnormalities detected at age} 5 to 6 years

Total CF-CT score at age 5-6 years was predictive of $\mathrm{FEV}_{1}$ (adjusted HR $\left.-0.02(-0.04--0.01) \mathrm{p}=0.006\right)$, FVC (adjusted HR $-0.02(-0.03--0.00), \mathrm{p}=0.044)$ and BMI for age Z-score decline (adjusted HR -0.02 $(-0.03-0.00) \mathrm{p}=0.017)$ for each point increase in CF-CT score (table 1 and eTables 2, 3, 4, 5 and 6).

Of all structural abnormalities, the extents of mucus plugging and trapped air were most strongly associated with $\mathrm{FEV}_{1}$ decline during the observation period. In adjusted analysis mucus plugging and trapped air were also significantly associated with $\mathrm{FVC}$ and $\mathrm{FEF}_{25-75} \mathrm{Z}$-scores (eTables 1, 2 and 3).

The extent of mucus plugging and bronchiectasis were the only chest CT abnormalities (apart from total CF-CT score) that were significantly predictive of BMI for age Z-score decline $(-0.10(-0.18--0.02)$ $\mathrm{p}=0.017$ and $-0.04(-0.08-0.01) \mathrm{p}=0.022$, respectively) in adjusted analysis (eTable 4$)$. Additionally, in a sensitivity analysis, out of all the sub-scores presence or absence of any mucus plugging on a CT scan at age 5-6 years best discriminated the difference in $\mathrm{FEV}_{1}$ rate of decline in the next 10 years of life. The rate of the decline in the presence and absence groups were $-0.08(-0.14--0.01) \mathrm{p}=0.017$ and $-0.00(-0.07-$ 0.06) $\mathrm{p}=0.903$, respectively (figure 3 and eTable 5).

\section{Percentage of future $\mathrm{FEV}_{1}$ explained by different structural abnormalities detected at age 5-6 years}

The predictive power of CT scores in young children is consistently stronger the more time elapses between initial CT scan and spirometry measurement in multivariate models (mucus plugging score at age 5-6 years explains only 3\% of additional variation in $\mathrm{FEV}_{1} \mathrm{Z}$-score at the same age but $>40 \%$ of additional variation 5 years later) while the opposite is true for $\mathrm{FEV}_{1} \mathrm{Z}$-score (at age 5 years it explains nearly $38 \%$ of additional variation in $\mathrm{FEV}_{1} 1$ year later but $<20 \%$ of additional variation 5 years later) (figure 4). A 

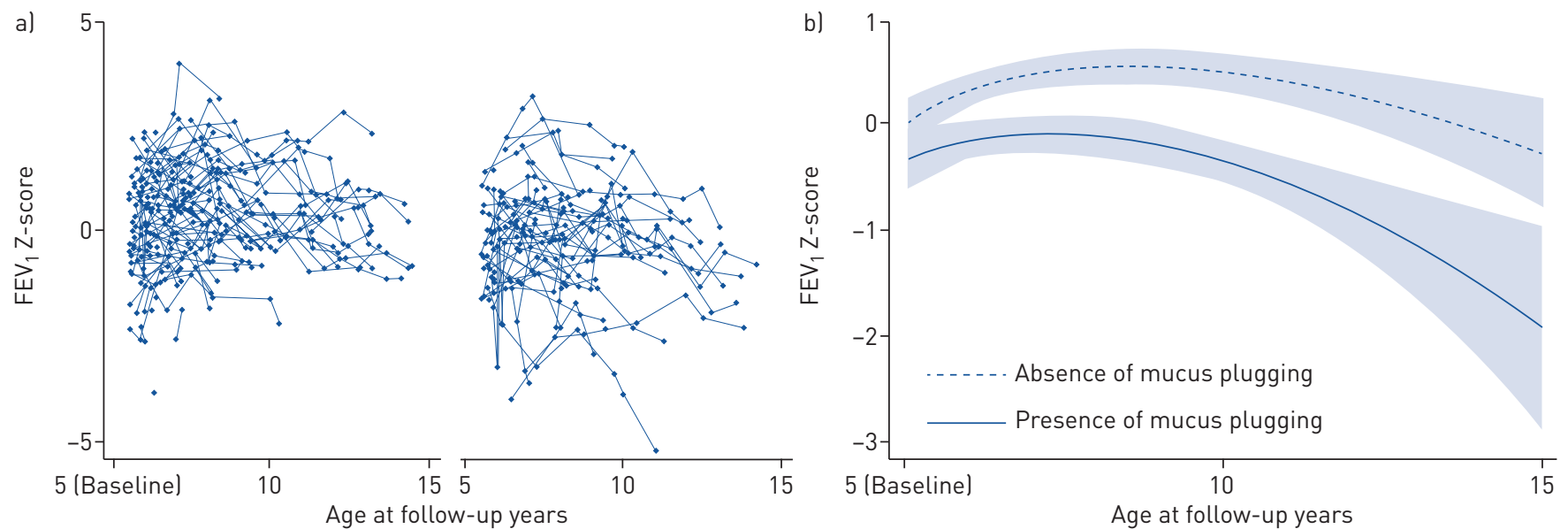

FIGURE 3 Forced expiratory volume in $1 \mathrm{~s}\left(\mathrm{FEV}_{1}\right)$ trajectories based on presence of mucus plugging on a computed tomography (CT) scan at age 5-6 years. a) Individual FEV ${ }_{1}$ trajectories of children that had no mucus plugging detected on a CT scan on the left and those that did on the right at age 5-6 years. b) Best fit fractional polynomial curves for both scenarios with corresponding $95 \%$ confidence intervals.

similar pattern is seen in other structural abnormalities. These results suggest that while $\mathrm{FEV}_{1}$ is a better predictor of future $\mathrm{FEV}_{1}$ in the short term, $\mathrm{CT}$ abnormalities such as mucus plugging and trapped air are better predictors of longer term $\mathrm{FEV}_{1}$. Spirometry measured $\mathrm{FEV}_{1}$ remains a better predictor of future $\mathrm{FEV}_{1}$ than bronchiactesis and bronchial wall thickening through the entire observation period.

\section{Discussion}

In this retrospective analysis of prospectively collected standardised data from the AREST CF cohort, we observed a strong relationship between structural lung disease in children $<6$ years of age and longer term clinical outcomes. We found that extent of mucus plugging and trapped air at age 5-6 years were strongly predictive of mean $\mathrm{FEV}_{1}$ decrease over the next 10 years. A unique and important observation was that CT scan evidence of mucus plugging at age 5-6 years was the only structural abnormality that showed a significant difference in rate of $\mathrm{FEV}_{1} \mathrm{Z}$-score decline between presence and absence groups.

AREST CF is a large paediatric CF cohort that is characterised by comprehensive prospective follow-up that includes annual or biennial CT scans from two Australian CF centres with comparable clinical management practices. We utilised data collected as a part of routine outpatient clinic visits after the end of the formal AREST CF early surveillance period (age 6 years), which enabled us to examine, in an unbiased manner, retrospective data collected prospectively that we hypothesised would predict long-term clinical outcomes. Results and conclusions in this study are robust and consistent across many different

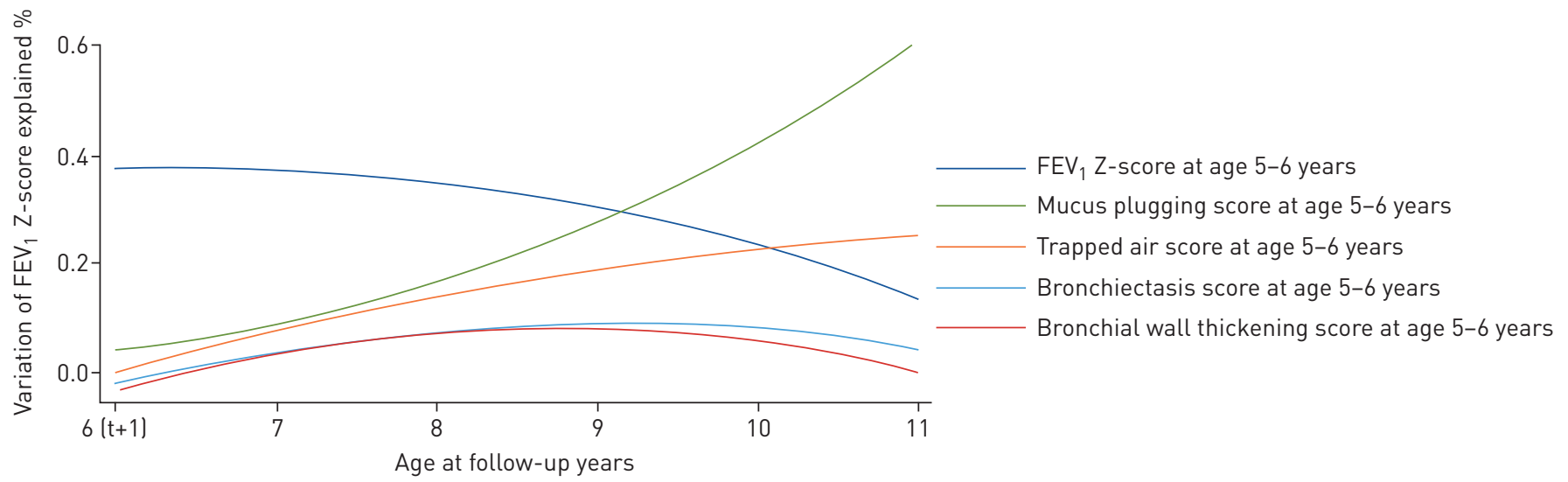

FIGURE 4 Percentage of forced expiratory volume in $1 \mathrm{~s}\left(\mathrm{FEV}_{1}\right)$ variation explained by different predictors at age 5-6 years in the next 5 years of life. Figure 3 represents adjusted $R^{2}$ in a series of multivariate cross-sectional regression models fitted to FEV $\mathrm{F}_{1}$ at age $6-11$ years as outcomes (one at the time). Base model $\mathrm{R}^{2}$ was first calculated for previously defined confounding variables. Additional $\mathrm{R}^{2}$ was then determined by adding the main exposure of interest to the base model and calculating the difference. Ages where models had data from $<30$ individuals were excluded from analysis due to instability of model estimates. 
modelling approaches and when using different outcome and predictor thresholds as was shown in our sensitivity analyses.

Other studies have been published that investigated relationships between CT and spirometry outcomes but assessed disease tracking comparison in a cross-sectional manner rather than longitudinal prediction of the future disease trajectory. The largest study published in 2006 [14], with 119 participants, compared the rate of progression of lung disease on serial CT scans and spirometry measurements in groups of children and adults and found them to be discordant, with bronchiectasis being the most reliable marker of disease progression. A study in 2004 [7] by the same group investigated paired high-resolution CT scans and spirometry tests in children of mean age 11 years at the first test and aimed to asses tracking of disease progression over a 2-year period. Their results are consistent with AREST CF data and demonstrated that mucus plugging and bronchiectasis are two components that progressed most, while additionally highlighting that spirometry remained stable during the same period.

One of the interesting and perhaps counter-intuitive findings in our study was that the predictive ability of mucus plugging and to a lesser extent trapped air was stronger than that of bronchiectasis which is considered a feature of irreversible lung damage. A possible explanation is that both trapped air and mucus plugging reflect an active disease process, with mucus plugging being an independent and persistent driver of inflammation and future airway damage, whereas bronchiectasis is an end-point reflecting prior pathological processes. Recent publications [22-24] support the hypothesis that airway surface dehydration due to cystic fibrosis transmembrane conductance regulator gene dysfunction leads to excessive mucus production which triggers chronic neutrophilic inflammation and structural lung damage [25]). Our data suggest that interventions that minimise mucus plugging are likely to have a significant effect on future lung disease trajectories.

Another important finding is the additional evidence for revising current guidelines for data collection in CF registries worldwide. Most registries currently only report respiratory data from age 6 years onwards, focusing on the age when spirometry starts to be routinely performed in CF centres. Even though many imaging studies have presented compelling evidence of early onset and progression of lung disease [7, 26-30], CT scan data are not routinely collected or included in registries. We have identified a time-period in children aged $\leqslant 6$ years during which a CT scan gives important clinical information and shows a clear relationship with later functional outcomes. Additionally, we found that structural sub-components are more predictive of functional trajectories 5 years later than lung function itself when measured at the same age. This suggests that besides CT providing additional important information regarding an individual's current clinical status, it can be used to identify children that are most at risk of worse clinical outcomes later in life. Therefore, a goal for CF centres could be the standardised acquisition of a single chest CT at age 5 years.

Due to limited CF populations available for traditional randomised controlled trials there is a need for new approaches to determine effectiveness of interventions as they become available. Our results provide a strong rationale for including CT outcomes at age 5-6 years in CF registries. Including childhood CT outcomes in CF registries would allow evaluation of the impact of new interventions in early life, before extensive lung damage has occurred, and the likely impact on adult lung function which is associated with long-term morbidity and mortality [31].

Low-dose radiation scans are considered safe and at age 5-6 years satisfactory images can be obtained without a general anaesthetic [32]. Further analysis of the AREST CF longitudinal data and outcomes from current intervention studies (COMBATCF NCT01270074 and SHIP-CT NCT02950883) that use CT as primary end-points will provide additional information regarding the CT outcomes of most value.

Two approaches have been published recently that might be more sensitive for detecting mild changes in lung structure than the CF-CT approach used in this study: PRAGMA-CF [33] and airway:artery ratio [6]. However, since these techniques involve new methods to image capture and analysis, there were insufficient CT images acquired that could be linked with long-term $\mathrm{FEV}_{1}$ follow-up measurements. A limitation of PRAGMA-CF is that because it is a hierarchical scoring system the independent effects on $\mathrm{FEV}_{1}$ of structural changes such as mucus plugging cannot be assessed. To increase the duration of follow-up, maximise the overall sample size and investigate the individual role of mucus plugging, we therefore used the CF-CT score in our analyses. Going forward, PRAGMA CF is a more discriminative marker of overall structural lung damage and a fully automated tool is in development and should be available in the near future. The PRAGMA CF score will provide clinicians with a validated outcome that assesses progression of mild disease that is not possible using spirometry. Therefore, clinicians should be able to better stratify high-risk patients for more intensive therapy.

We acknowledge that validation of our results in an independent cohort should be undertaken. In the meantime, we believe that our data are likely to be representative [34] and difficult to replicate in the near 
future due to sample size requirements and the unique nature of the AREST CF programme. We also acknowledge that while this data is based on a group of children and is useful for stratification of groups at risk, use of absolute Brody CF-CT scores as a clinical tool for individual screening should not be over-interpreted.

In conclusion, both bronchiectasis and non-bronchiectatic lung abnormalities in young children with CF are important markers of disease severity and predictors of future lung disease progression. The extent of mucus plugging at age 5 to 6 years provides a simple stratification tool that can identify children at increased risk of poor outcomes in later life and is an early indicator of the effectiveness of interventions in very young children that have disease modifying potential.

Acknowledgments: We would like to thank Yuliya Karpievitch for providing a statistical review of this manuscript.

Conflict of interest: L. Turkovic has nothing to disclose. D. Caudri has nothing to disclose. T. Rosenow reports grants from National Health and Medical Research Council, during the conduct of the study. In addition, Dr. Rosenow has a patent PCT/AU2016/000079 issued to the Australian Patent Office. O. Breuer has nothing to disclose. C. Murray has nothing to disclose. H.A.W.M. Tiddens reports other from Roche and Novartis, grants from CFF, Vertex, Chiesi, Vectura and NCFS, outside the submitted work. In addition, he has a patent PRAGMA-CF scoring system issued and is head of the Erasmus MC-Sophia Children's Hospital core laboratory LungAnalysis. F. Ramanauskas has nothing to disclose. S.C. Ranganathan has nothing to disclose. G.L. Hall has nothing to disclose. S.M. Stick has nothing to disclose.

Support statement: Cystic Fibrosis Foundation (http://dx.doi.org/10.13039/100000897), National Health and Medical Research Council (http://dx.doi.org/10.13039/501100000925). Funding information for this article has been deposited with the Crossref Funder Registry.

\section{References}

1 Riordan JR, Rommens JM, Kerem B, et al. Identification of the cystic fibrosis gene: cloning and characterization of complementary DNA. Science 1989; 245: 1066-1073.

2 Mayer-Hamblett N, Ramsey BW, Kronmal RA. Advancing outcome measures for the new era of drug development in cystic fibrosis. Proc Am Thorac Soc 2007; 4: 370-377.

3 Rosenfeld M. An overview of endpoints for cystic fibrosis clinical trials: one size does not fit all. Proc Am Thorac Soc 2007; 4: 299-301.

4 Koch C, McKenzie SG, Kaplowitz H, et al. International practice patterns by age and severity of lung disease in cystic fibrosis: data from the Epidemiologic Registry of Cystic Fibrosis (ERCF). Pediatr Pulmonol 1997; 24: $147-154$.

5 Singer RB. Cystic fibrosis mortality: registry data of cystic fibrosis. J Insur Med 1997; 29: 233-239.

6 Bell SC, Bye PT, Cooper PJ, et al. Cystic fibrosis in Australia, 2009: results from a data registry. Med J Aust 2011; 195: 396-400.

7 de Jong PA, Nakano Y, Lequin MH, et al. Progressive damage on high resolution computed tomography despite stable lung function in cystic fibrosis. Eur Respir J 2004; 23: 93-97.

8 Kerem E, Viviani L, Zolin A, et al. Factors associated with $\mathrm{FEV}_{1}$ decline in cystic fibrosis: analysis of the ECFS patient registry. Eur Respir J 2014; 43: 125-133.

9 Mott LS, Park J, Murray CP, et al. Progression of early structural lung disease in young children with cystic fibrosis assessed using CT. Thorax 2012; 67: 509-516.

10 Sly PD, Brennan S, Gangell C, et al. Lung disease at diagnosis in infants with cystic fibrosis detected by newborn screening. Am J Respir Crit Care Med 2009; 180: 146-152.

11 Stick SM, Brennan S, Murray C, et al. Bronchiectasis in infants and preschool children diagnosed with cystic fibrosis after newborn screening. J Pediatr 2009; 155: 623-628.

12 Martínez-García MA, Perpiñá-Tordera M, Román-Sánchez P, et al. Quality-of-life determinants in patients with clinically stable bronchiectasis. Chest $2005 ; 128: 739-745$.

13 Tepper LA, Utens EM, Caudri D, et al. Impact of bronchiectasis and trapped air on quality of life and exacerbations in cystic fibrosis. Eur Respir J 2013; 42: 371-379.

14 de Jong PA, Lindblad A, Rubin L, et al. Progression of lung disease on computed tomography and pulmonary function tests in children and adults with cystic fibrosis. Thorax 2006; 61: 80-85.

15 Tepper LA, Caudri D, Rovira AP, et al. The development of bronchiectasis on chest computed tomography in children with cystic fibrosis: can pre-stages be identified? Eur Radiol 2016; 26: 4563-4569.

16 Young K, Aspestrand F, Kolbenstvedt A. High resolution CT and bronchography in the assessment of bronchiectasis. Acta Radiol 1991; 32: 439-441.

17 Standardization of Spirometry, 1994 Update. American Thoracic Society. Am J Respir Crit Care Med 1995; 152 : $1107-1136$.

18 Miller MR, Hankinson J, Brusasco V, et al. Standardisation of spirometry. Eur Respir J 2005; 26: 319-338.

19 Quanjer PH, Stanojevic S, Cole TJ, et al. Multi-ethnic reference values for spirometry for the 3-95-yr age range: the global lung function 2012 equations. Eur Respir J 2012; 40: 1324-1343.

20 Pellegrino R, Viegi G, Brusasco V, et al. Interpretative strategies for lung function tests. Eur Respir J 2005; 26 : 948-968.

21 WHO Multicentre Growth Reference Study Group. WHO Child Growth Standards based on length/height, weight and age. Acta Paediatr Suppl 2006; 450: 76-85.

22 Mall MA. Unplugging mucus in cystic fibrosis and chronic obstructive pulmonary disease. Ann Am Thorac Soc 2016; 13: Suppl. 2, S177-S185.

23 Mall MA, Stahl M, Graeber SY, et al. Early detection and sensitive monitoring of CF lung disease: prospects of improved and safer imaging. Pediatr Pulmonol 2016; 51: S49-S60. 
24 Montgomery ST, Mall MA, Kicic A, et al. Hypoxia and sterile inflammation in cystic fibrosis airways: mechanisms and potential therapies. Eur Respir J 2017; 49: 1600903.

25 Esther CR Jr, Muhlebach MS, Ehre C, et al. Mucus accumulation in the lungs precedes structural changes and infection in children with cystic fibrosis. Sci Transl Med 2019; 11: eaav3488.

26 Brody AS, Klein JS, Molina PL, et al. High-resolution computed tomography in young patients with cystic fibrosis: distribution of abnormalities and correlation with pulmonary function tests. J Pediatr 2004; 145: 32-38.

27 Helbich TH, Heinz-Peer G, Fleischmann D, et al. Evolution of CT findings in patients with cystic fibrosis. AJR Am J Roentgenol 1999; 173: 81-88.

28 Kongstad T, Green K, Buchvald F, et al. Association between spirometry controlled chest CT scores using computer-animated biofeedback and clinical markers of lung disease in children with cystic fibrosis. Eur Clin Respir J 2017; 4: 1318027.

29 Loeve M, Hop WC, de Bruijne M, et al. Chest computed tomography scores are predictive of survival in patients with cystic fibrosis awaiting lung transplantation. Am J Respir Crit Care Med 2012; 185: 1096-1103.

30 Santamaria F, Grillo G, Guidi G, et al. Cystic fibrosis: when should high-resolution computed tomography of the chest be obtained? Pediatrics 1998; 101: 908-913.

31 Hulzebos EH, Bomhof-Roordink H, van de Weert-van Leeuwen PB, et al. Prediction of mortality in adolescents with cystic fibrosis. Med Sci Sports Exerc 2014; 46: 2047-2052.

32 Kuo W, Ciet P, Tiddens HA, et al. Monitoring cystic fibrosis lung disease by computed tomography. Radiation risk in perspective. Am J Respir Crit Care Med 2014; 189: 1328-1336.

33 Rosenow T, Oudraad MC, Murray CP, et al. PRAGMA-CF. A quantitative structural lung disease computed tomography outcome in young children with cystic fibrosis. Am J Respir Crit Care Med 2015; 191: 1158-1165.

34 Martin B, Schechter MS, Jaffe A, et al. Comparison of the US and Australian cystic fibrosis registries: the impact of newborn screening. Pediatrics 2012; 129: e348-e355. 